\title{
Facklamia hominis
}

National Cancer Institute

\section{Source}

National Cancer Institute. Facklamia hominis. NCI Thesaurus. Code C122307.

A species of gram-positive, catalase-neg ative, facultatively anaerobic, coccus-shaped bacteria in the family Firmicutes. F. hominis is a normal part of the female genital tract flora but may act as an opportunistic pathogen. 